Este artículo expone el proceso de investigación realizado en el marco de la pasantía correspondiente a la Convocatoria Jóvenes Investigadores 2008, abierta por el Instituto Colombiano para el Desarrollo de la Ciencia y la Tecnología Francisco José de Caldas - COLCIENCIAS -, en la que resultaron seleccionadas las autoras del documento. El tema central es la consolidación de un Observatorio de Medios Escolares para el Distrito Capital, en la Facultad de Ciencias de la Comunicación de la Universidad Minuto de Dios con el ánimo de generar procesos de investigación entorno a las diversas prácticas, procesos pedagógicos y comunicativos que reconozcan a los medios escolares de comunicación como una herramienta para potenciar las condiciones de desarrollo tanto de las instituciones educativas como de los contextos y agentes que las configuran.

Palabras clave: medios escolares, observatorios de medios, procesos pedagógicos, convivencia, participación y ciudadanía.

Synthesis:

This paper presents the research process as part of the internship for the 2008 Open Call for Young Researcher by the Institute for the Development of Science and Technology Francisco José de Caldas - COLCIENCIAS - in which the authors were selected the document. The central theme is the consolidation of a Media Centre for the Capital District School in the Faculty of Communication Sciences at the University Minuto de Dios, with the aim of generating research processes around various practices, pedagogical and communicative processes that recognize school means of communication as a tool to enhance the conditions for development of both educational institutions and contexts that shape agents.

Key Words: scholar, media observatories, pedagogical processes, coexistence, participation and citizenship

\title{
Observatorio de medios escolares en el Distrito Capital: miradas sobre el quehacer propio
}

Elssy Yamile Moreno Comunicadora Social-Periodista, Magíster en

Desarrollo educativo y social e-mail: elyamope@yahoo.com

Ibeth Johana Molina Comunicadora Social-Periodista, Magíster en

Desarrollo educativo y social

e-mail: ibethmol@gmail.com Fecha de recepción: 01-08-2010 Fecha de aprobación: 16-09-2010

\section{Introducción}

"El derecho al conocimiento es también el derecho a la producción de conocimientos, a la recuperación de conocimientos, a la comunicación de conocimientos. Los sistemas educativos pueden, en este sentido, aprender muchísimo de sí mismos".

Daniel Prieto Castillo 


\section{La idea de esta investigación nace del interés} por fortalecer los procesos de desarrollo humano y social. La educación como proceso de constitución del sujeto, es uno de sus pilares fundamentales. La escuela es uno de los escenarios en los que transita dicho proceso; en la actualidad este escenario cuenta con una oferta mediática de interés para los educandos (prensa, radio, televisión y las TIC).

Así mismo, en la escuela es frecuente encontrar problemáticas como la violencia, el manejo inadecuado de los conflictos y el desconocimiento de actores y factores del contexto en que está inmersa. Esta situación plantea retos y necesidades que deben abordarse desde diferentes disciplinas.

Actualmente existen múltiples observatorios de medios de comunicación en el país y en la región, además con diversos enfoques. En su mayoría realizan un monitoreo y posterior análisis de contenido de los mismos; sin embargo, en Bogotá, los medios escolares no han sido objeto de este tipo de procesos.

El propósito de este proyecto, tal como se planteó en la propuesta presentada al Instituto Colombiano para el Desarrollo de la Ciencia y la Tecnología Francisco José de Caldas -COLCIENCIAS-, es generar procesos de investigación en torno a las diversas prácticas pedagógicas y comunicativas que incluyen o podrían incluir a los medios escolares de comunicación, como herramienta para potenciar las condiciones de desarrollo tanto de las instituciones educativas como de los contextos en que éstas se encuentran inmersas.

Es necesario aclarar que en este caso, cuando se habla de procesos de investigación no se hace referencia únicamente a los adelantados por el equipo base de trabajo, sino también a aquellos desarrollados por los estudiantes y docentes de pregrado y posgrado de la Facultad de Ciencias de la Comunicación de UNIMINUTO, quienes se vinculan a cada una de las fases del proyecto y a los de los colectivos de comunicación de las instituciones educativas participantes.

De otro lado, cuando se habla de observación la mirada no sólo se dirige hacia el monitoreo de los medios, ni al análisis de contenido sobre los mismos; que es lo que suele hacerse tradicionalmente, sino a la construcción de herramientas que faciliten la evaluación y definición tanto de los lineamientos metodológicos como de la orientación pedagógica de las prácticas mediáticas en entornos escolares, para de esta manera contribuir al fortalecimiento de las relaciones escuela - entorno.

Se trata entonces de generar en los participantes competencias para reflexionar, comprender y si se requiere, transformar tanto el sentido como las prácticas relacionadas con los medios escolares de comunicación y sus contextos. En este sentido, se formularon tres preguntas que orientaron la investigación y seguirán orientando el quehacer del Observatorio, estas son: ¿Cómo se están asumiendo los procesos comunicativos en la escuela? ¿Qué tipo de prácticas sugiere la inclusión de los medios en este escenario? y icómo se re-significan o gestionan los procesos de desarrollo a partir de la existencia de estos recursos en el contexto educativo?

\section{Descripción metodológica}

Inicialmente se plantearon dos actividades generales, la primera era la realización del Primer Concurso de Medios Escolares, que buscaba un acercamiento a las creaciones mediáticas en las instituciones educativas 
del Distrito Capital y la segunda era el análisis concreto de experiencias de medios escolares para determinar cómo contribuyen al fortalecimiento de los procesos pedagógicos, la convivencia y la construcción de ciudadanía en la escuela.

Posteriormente, con la vinculación de estudiantes de pregrado y posgrado de la Facultad de Ciencias de la Comunicación, es decir, con la consolidación de los semilleros de investigación en el proyecto (a través de dos modalidades: trabajo de clase y asistencia de investigación), se plateó una serie de actividades adicionales que permitieran el logro de los propósitos enunciados anteriormente. Estas fueron: realización del Primer Concurso de Medios Escolares, acercamiento y análisis de las experiencias correspondientes a los productos entregados en el concurso; revisión de categorías como medios escolares, observatorios de medios y comunicación y educación; revisión de legislación concerniente a medios escolares en el Distrito Capital; realización de los perfiles de las organizaciones que trabajan el tema en el Distrito y análisis de las convocatorias realizadas durante los últimos seis años. Vale la pena aclarar que este artículo se centra únicamente en la fundamentación teórica del proyecto, la revisión de antecedentes empíricos y el planteamiento de la propuesta macro; sin embargo, se hará una enunciación de los principales resultados obtenidos a la fecha tras la implementación de la propuesta.

De lo que se trata entonces es de la construcción del proyecto de investigación a poner en marcha en la Facultad de Ciencias de la Comunicación, denominado: "Observatorio de medios escolares en el Distrito Capital". A continuación planteamos las posturas teóricas desde las que se construye dicho proyecto.

\section{Aproximaciones teórico-conceptuales}

Este proyecto se soporta teóricamente en tres categorías: comunicación/educación, observatorios de medios y medios escolares; las tres constituyen los lugares de partida, pero también de llegada, en tanto se busca reconocer la situación actual de diversos entornos educativos para luego buscar potenciarlos como alternativas de desarrollo.

En este sentido, la categoría de comunicación para el desarrollo es la categoría macro en la que se inscribe este proyecto, sin embargo, es en la relación comunicación/educación, como campo de estudio, en donde se asienta, pues consideramos que ésta, puede y debe contribuir a la gestión del desarrollo, más aún en el caso de la escuela como uno de los escenarios educativos más significativos en el proceso de construcción de los sujetos.

Se trata entonces de realizar un ejercicio individual y colectivo de observación de los procesos propios y ajenos, generando procesos de interaprendizaje, de diálogo, de consensos y disensos, que contribuyan a la gestión del desarrollo. Es decir, este proyecto solo puede realizarse en la medida en que los participantes tengan el interés y la voluntad para adentrarse en los procesos comunicativos que ocurren en la escuela.

\section{Comunicación/Educación}

"Es académicamente posible y políticamente deseable que se considere la interrelación entre comunicación y educación como un nuevo campo de intervención social y de investigación científica en América Latina. Este reconocimiento significa que ni la comunicación ni la educación han alcanzado aisladamente a atender determinadas necesidades o resolver determinados 
problemas del 'estar con-en el mundo' y de la construcción de relaciones igualitarias y democráticas entre los seres humanos" ${ }^{\prime 1}$.

Esta afirmación de Ismar de Olivera nos hace pensar que la interrelación entre Comunicación y Educación, es necesaria y pertinente en el contexto latinoamericano, pero aún es incipiente. Como lo desarrollaremos en este apartado, es un campo de estudio que está en proceso de construcción pues desde hace poco menos de 20 años se habla del mismo, pero que como tal, tiene grandes dificultades y potencialidades, puesto que se ha alimentado desde diversas posturas teóricas, críticas y políticas.

A manera de ejemplo, podría decirse que para algunos (educadores, educandos, comunicadores) aún hoy en día la relación comunicación/educación no es otra cosa que la incursión del medio de comunicación (prensa, radio, televisión e internet) en la escuela, sin importar el sentido de dicha acción. Como bien lo menciona Jorge Huergo en varios de sus textos, las dos disciplinas han sido víctimas de reduccionismos tecnicistas, en tanto la primera suele equipararse al medio y la segunda a la escuela.

En esta misma línea Mario Kaplún plantea que la relación entre comunicación y educación tenía lugar desde tres posturas: educación que hace énfasis en los contenidos, educación que enfatiza en los efectos y educación que pone énfasis en el proceso; la primera se refiere al modelo lineal de la comunicación, la segunda se basa en el mismo proceso (emisor-canal-receptor) pero reconoce la retroalimentación del receptor; y la

${ }^{1}$ OLIVEIRA SOARES, Ismar de. "La Educomunicación como desafío para nuestras organizaciones", En: Revista Aradü, No. 41. Quito: Ecuador. tercera se basa, según Kaplún, en la transformación de las personas y de las comunidades, teniendo en cuenta la interacción entre el emisor y el receptor. A partir de estas concepciones y las de otros autores como Jesús Martín Barbero, Pedro Gómez, quienes proponen la relación entre comunicación y educación como un proceso de interrelación entre el sujeto y los medios. Consideramos que la postura planteada por Gómez no reconoce ni los otros escenarios educativos ni los procesos comunicativos no mediáticos.

Son varios los autores que dedican o han dedicado parte de su tiempo e intereses académicos al campo en construcción de la comunicación/educación. Entre los latinoamericanos encontramos a Mario Kaplún y Paulo Freire, Rosa María Alfaro, Daniel Prieto Castillo, Guillermo Orozco, Jesús Martín Barbero, Jorge Huergo, Carlos Valderrama, Humberto Cubides, Ismar de Oliveira. ${ }^{2}$ Quisiéramos reconocer los aportes más significativos de algunos de ellos con el propósito de realizar una mirada más compleja sobre los procesos comunicativos que ocurren en la escuela, inicialmente en el Distrito Capital.

Sin temor a equivocarnos podríamos decir que Jorge Huergo quizá es el autor latinoamericano que más se ha interesado y ha estudiado el campo a profundidad.

${ }^{2}$ Para mayor información el lector puede consultar: OLIVERIA, Soares Ismar de. Sociedad de la Información o de la Comunicación. Sao Paulo: Cidade Nova,1996.; KAPLÚN, Mario. A la educación por la comunicación: La práctica de la comunicación educativa, Chile: Unesco/ Orelac. 1992; FREIRE, Paulo. Pedagogía del Oprimido, Madrid: Siglo XXI Editores.1995; PRIETO C. Daniel. La comunicación en la educación, Buenos Aires: Ed. La Crujía Ciccus. 1999; VALDERRAMA, Carlos Eduardo et. Al. Comunicación-Educación, coordenadas abordajes, travesías, Bogotá: Universidad Central. 2000; y OROZCO, Guillermo. "Desordenamientos Educativos en el Ecosistema Comunicacional" En: Comunicación Educativa en la Sociedad de la Información, Madrid: UNED. 2003. 
En su tesis de maestría titulada "Hacia una genealogía de la comunicación/educación. Rastreo de algunos anclajes político-culturales", publicada en el año 2005, continúa sus trabajos anteriores (casi 30 años de prácticas educativas e investigativas) y desentraña los orígenes de la interrelación entre comunicación y educación en los discursos de carácter teórico, cómo él mismo lo afirma, desde una perspectiva genealógica y fundacional; sin desconocer que estos discursos han incidido en las prácticas sociales y profesionales de muchos educadores y comunicadores en América Latina.

Los elementos más significativos que plantean la discusión de Huergo son la instrumentalización de las dos disciplinas (sus reduccionismos) y la separación de los procesos educativos-comunicativos del contexto político-cultural. Así mismo reconoce factores como el desarrollismo que impregnó a los pueblos y a los gobiernos de la necesidad de progreso (aumento de capital y tecnologías, para el caso, tecnologías educativas con fines expansionistas) y las promesas no cumplidas de la modernidad (escuela como educadora del buen ciudadano) que influyeron substancialmente en la manera que se empezó a construir el campo, en tanto se desconocen las crisis, los conflictos, las dificultades económicas, todos factores que influyen en la manera en que los sujetos se relacionan.

El tema de la comunicación intersubjetiva como objeto de análisis en la construcción del campo también es relevante en el trabajo de Huergo; él cita a otros autores como Francisco Gutiérrez y afirma que: "El autor percibe la articulación entre las dos dimensiones de las transformaciones culturales: la dimensión objetiva

${ }^{3}$ HUERGO, Jorge. Hacia una genealogía de Comunicación/Educación. Rastreo de algunos anclajes Político-Culturales. Argentina: Universidad Nacional de La Plata. 2005. y la dimensión subjetiva"3 al respecto Gutiérrez dice "La civilización moderna con sus medios técnicos de transporte (trenes, automóvil, avión), sus medios de comunicación (prensa, radio, cine, TV), en fin, con sus medios mecánicos y hasta electrónicos de interrelación está ofreciendo al hombre nuevas formas de percibir, de intuir, sentir y pensar. (...) El cambio de percepción implica el cambio de mentalidad" ${ }^{\prime}$.

Rosa María Alfaro por su parte cuestionó aquellas propuestas que pusieron el acento en la crítica a los medios como culpables de la alienación (teoría de los efectos y la dependencia), desconociendo el valor de los mismos y las posibilidades de interrelación entre comunicación y educación. En sus textos evidencia la tensión entre quienes satanizan a los medios (padres, educadores, audiencias) y quienes producen el contenido de los medios, pues con frecuencia desconocen su influencia sobre las audiencias.

Consideramos que otro de los tantos aportes valiosos de Alfaro a este proyecto se da en relación con la manera en que asume la educación, como una construcción colectiva que se da en diversos lugares con particularidades sociales, económicas, políticas y culturales. En este sentido, los medios de comunicación pueden verse también como un escenario educativo, mediado y mediador de los diferentes contextos de los sujetos, que le brindan alternativas para pensar sus procesos de desarrollo; al respecto dice:

"Los propios sistemas de mediación son útiles para reconocerse y conocer a otros, para provocar procesos de aprendizaje, para dialogar con

${ }^{4}$ GUTIÉRREZ, Francisco. "EI Lenguaje Total. Pedagogía de los medios de comunicación”. Buenos Aires: Hvmanitas. 1973. En: HUERGO, Jorge. Ibid. 
sus contrarios con los simplemente diferentes. No hay educación posible sin comunicación, la que posibilita significativas interacciones de enseñanzas y aprendizajes (...) Hoy podemos afirmar que los sujetos sí conocen su realidad y de manera vital (...) el desarrollo, sí se conecta a estas dinámicas [las de la realidad] sin frivolizarlas, tiene más capacidad educativa y de cambio que el retrato racionalizado de una realidad deprimente, oscura y sin salidas [planteado por Freire]. Cabe señalar las nuevas propuestas educativas que se están aportando en el campo de la relación entre consumidores y medios, utilizando para ello como agentes activos a instituciones y profesionales de la escuela, las ONG y las propias familias.

En todos los casos, por lo tanto, cada proyecto de desarrollo debe contener una estrategia comunicativa precisa que defina las relaciones a construir, los métodos, sus etapas, sus posibles conflictos y soluciones, sustentados en diagnósticos no sólo sociales, sino también comunicativos, abordando la intersubjetividad" ${ }^{5}$.

Como vemos hasta este punto, la interrelación entre comunicación y educación debe reconocer las diferentes dimensiones del desarrollo, para poder potenciarlas en cualquier escenario, para este caso, en la escuela.

\section{Medios escolares, herramientas para el desarrollo educativo y social}

Los medios de comunicación en entornos educativos son un recurso cada vez más necesario en las prácticas

5. ALFARO, Rosa María. "Lo educativo en la comunicación”, En: La comunicación como relación para el desarrollo, Lima: Calandria. 1993, págs. 11-12. pedagógicas de las instituciones educativas, por eso, han sido motivo de algunos estudios o revisiones desde entidades como el IECO (Instituto de Estudios en Comunicación y Cultura) de la Universidad Nacional, el IDEP (Instituto para la Investigación Educativa y el Desarrollo Pedagógico), de la Secretaría de Educación del Distrito (SED), el IESCO (Instituto de Estudios Sociales Contemporáneos) de la Universidad Central, entre otras. El tema también se ha abordado desde investigaciones de pregrado y posgrado, tratando de reconocer algunas prácticas de inclusión de los medios en la escuela.

A continuación, presentamos algunos hallazgos de estas experiencias, que son fundamentales al momento de consolidar alguna propuesta para pensar los medios en entornos educativos. El primer estudio consultado se denomina "Comunicación y Escuela: Orientaciones para la incorporación, usos y apropiación de los medios de comunicación en las Instituciones Educativas de Bogotá", producido en el 2004 por la Secretaría de Educación del Distrito, y el grupo de investigación Red, del IECO. En dicho estudio, se plantean unas orientaciones para la incorporación, usos y apropiación de los medios en la escuela, como eslabón en la generación de política pública acerca de la comunicación/educación en Bogotá, analizando diversas tendencias de inclusión de los medios y las TIC en entornos escolares. Entre ellas destacamos: a) la incorporación del medio al currículo, como asignatura específica o como eje transversal, que articule las $\mathrm{TIC}$, las estéticas y las narrativas propias de los medios, de tal forma que se produzcan nuevas maneras de interpretar o interpelar los saberes que circulan en la escuela, b) educación en y con los medios, a partir de proyectos comunicativos que planteen una apuesta creativa de comunicación, generen saberes y experiencias sociales distintas a las que transitan por los medios comerciales, pero también partiendo de proyectos comunicativoeducativos que incorporen los medios en los procesos 


\section{Aprendizajes}

de educación para la emancipación de los sujetos y la construcción de tejido social, desde propuestas que indaguen por la generación de nuevos saberes con un sentido pedagógico, político y cultural. Además, propone incorporar los medios al currículo escolar a partir de tres horizontes: la alfabetización, la recepción crítica y el uso de los medios desde una apuesta crítica y transformadora en relación con el modelo curricularb.

De igual manera, el estudio identificó tres perspectivas de incorporación, usos y apropiación de los medios y las tecnologías de la información y la comunicación en las instituciones educativas de la ciudad:

La primera es la perspectiva informacional o instrumental, que reduce los medios de comunicación a medios de enseñanza, generando escisiones entre prácticas comunicativas y educativas, "no pasan de ser más que prácticas puntuales que superponen los saberes escolares con el saber de sentido común que se posee sobre los medios, impidiendo que los medios se articulen a la vida escolar; por tanto, se niega la posibilidad de preguntarse desde perspectivas pedagógico-políticas acerca del sentido de los medios en la escuela y de sus posibilidades para dinamizar formas abiertas de comunicación con el mundo y con sus contextos locales"7.

La segunda perspectiva es la comunicación social en la escuela, "se caracteriza por llevar a cabo un estudio

7. Ibid, pág. 47.

6. ALCALDÍA MAYOR, SED. Comunicación y escuela: Orientaciones para la incorporación, usos y apropiación de los medios de comunicación en las Instituciones Educativas de Bogotá. Bogotá: Secretaría Educación del Distrito. 2005. 
De igual manera, en el 2005 el IDEP (Instituto para la Investigación Educativa y el Desarrollo Pedagógico) a partir de la publicación "A-prender los medios. Experiencias pedagógicas" expone siete experiencias que participaron en una convocatoria cuyo objeto fue "seleccionar, apoyar, socializar y financiar proyectos de innovación pedagógica orientados a desarrollar las competencias comunicativas de los estudiantes de educación básica y media de las instituciones de Bogotá, D.C., a través del uso pedagógico de los medios de comunicación"10. En dicho texto, podemos encontrar experiencias que integran medios como la televisión (desde la lectura y recepción crítica de contenidos), el video (desde la producción), la radio (desde la recepción crítica pero también desde la producción) y el periódico mural o impreso. Estas experiencias tienen un valor agregado desde la perspectiva de corte pedagógicocrítico que plantea el estudio del IESCO y la SED, desde diferentes alternativas de articulación entre los modelos curriculares, los medios, el consumo crítico de contenidos y el fortalecimiento de competencias comunicativas; propiciando formas creativas de apropiación y construcción de saberes desde el ámbito educativo. Esta publicación hace énfasis en la argumentación teórica que valida cada experiencia, así como los aprendizajes, conclusiones y desarrollos metodológicos propios de cada institución educativa participante.

Otro antecedente que nos remite a pensar el papel de los medios en la escuela lo encontramos en el documento "Radio escolar: comunicación, conflictos y ciudadanías" de César Augusto Rocha. Este ejercicio investigativo que duró tres años en un colegio de Bogotá, buscó, a partir de un proceso comunicativo, construir aprendizajes

10. ALCALDÍA MAYOR, IDEP. A-prender los medios. Bogotá: Instituto para la Investigación Educativa y el Desarrollo Pedagógico. 2005, pág. 2. entre los diferentes actores de la comunidad educativa, sobre el manejo de conflictos a través de la radio escolar, re-significando las mediaciones que pueden generarse a partir de los medios, desde una reflexión pedagógica que contribuya al campo teórico de la relación comunicación - educación.

Esta investigación buscó dar a conocer diferentes explicaciones intencionales de los actores escolares a la hora de hablar de su cotidianidad, con el fin de gestionar pedagógicamente los conflictos y problemáticas propias de la institución educativa y del territorio en el cual se encontraba inmersa. La radio escolar se constituyó como un espacio, pero a la vez como una herramienta que facilitó el interaprendizaje entre los actores escolares a partir de las diferentes reflexiones y análisis realizados por los participantes.

Luego de la revisión de estas experiencias, podemos concluir que la inclusión de los medios en la escuela más que una alternativa de modernización o una innovación en las tecnologías educativas es una necesidad en tanto éstos [los medios] se han convertido en herramientas que intervienen en los procesos primarios de socialización para los niños y jóvenes, quienes se encuentran inmersos en lo que Martín Barbero ha denominado "ecosistemas comunicativos", es decir, nuevas tecnicidades comunicativas mediadas por el avance de las TIC en un mundo cada vez más globalizado, que producen nuevas formas de apropiación y generación de conocimientos. De ahí la importancia y necesidad de reconocer a los medios de comunicación escolares como eslabones mediadores de imaginarios, identidades y nuevas formas de representación de los jóvenes.

"La escuela debe interactuar, no tanto con los medios, debe interactuar a través de ellos con los 'nuevos 
campos de experiencia' en que hoy se procesan los cambios, es decir, las hibridaciones entre ciencia y arte, entre cultura escrita y audiovisual, y la reorganización de los saberes en los flujos y redes por los que hoy se moviliza la información, el trabajo, la creatividad"11.

\section{Observatorios de medios, ¿una moda? ¿Una propuesta política?}

Los observatorios de medios se han constituido como un espacio de veeduría de acción civil por parte de las audiencias (académicos e investigadores de instituciones universitarias y algunas $O N G$ ) frente a los medios masivos de comunicación y los mensajes que circulan a través de éstos, a partir de elementos como el análisis de la relación entre industria mediática y el mercado, las agendas temáticas que se constituyen desde los contenidos, las preferencias de consumo por parte de las audiencias, los marcos éticos y legales de regulación que determinan las prácticas mediáticas, pero también de los contenidos y el impacto que éstos producen en los imaginarios y en las prácticas sociales. "La crítica al funcionamiento de los medios recupera visibilidad no sólo centrada en su poder monopólico sino en el rol y poder que van adquiriendo en la forja de opinión pública y culturas políticas en cada país"12.

Rosa María Alfaro, por su parte nos habla de la emergencia de mecanismos como los Observatorios de Medios que permiten generar otras formas de denunciar algunas prácticas mediáticas de carácter masivo que minaron de incertidumbre a las audiencias

\footnotetext{
11. MARTÍN-BARBERO, Jesús. "La educación en el ecosistema comunicativo”, En: Revista Comunicar No. 13. 1999, págs: 13-21. En línea.

12. ALFARO, Rosa María. Observatorios de medios: avances, limitaciones y retos. ¿Una nueva conciencia crítica o una ruta de cambio? Lima: Calandria. 2005, pág. 3.
}

y generaron fuertes críticas frente al papel de los medios en el continente, dando lugar a diferentes dinámicas sociales e institucionales que buscan velar por la calidad de los contenidos y el accionar ético de los periodistas y los medios en general en América Latina:

\begin{abstract}
"Desde los observatorios se va percibiendo a los medios como un lugar clave de construcción de ciudadanía que había que disputar o equilibrar. Otro componente motivador estuvo en el crecimiento de una asociación peligrosamente construida entre política, medios y mercado, lo que ya venía generando fenómenos de corrupción en la medida que primaba el negocio como alianza básica. Así los medios estaban o podían estar comprometidos en negocios de cualquier tipo con el Estado, partidos o intereses privados, cuyas evidencias están hoy a la orden del día dada su envergadura. (...) Igualmente, se observaba un proceso degenerativo en el campo del entretenimiento con ofertas simplistas sin perspectiva ética y de calidad, las que incomodaban a un gran sector de la ciudadanía. Todo lo cual produjo un gran descontento entre los públicos quienes se daban cuenta que construían opiniones con escasa y pobre información, descriptiva y sin análisis"13.
\end{abstract}

En sentido estricto, los observatorios de medios se convierten en un escenario de reflexión, investigación y socialización frente al quehacer de los medios masivos de comunicación a partir del análisis de la información, como una posibilidad de denuncia, de exigencia y de control de calidad por parte de las audiencias frente a las propuestas mediáticas, pero en algunos casos, estos observatorios se convierten también en

13. Ibid, pág. 4. 
un espacio de formación y de consumo crítico a partir de procesos educativos, bien sea con asociaciones o redes conformadas por las audiencias.

En cuanto a las temáticas, la mayoría de los observatorios de medios en Latinoamérica tienen que ver con el derecho a la información, la calidad de la información, el periodismo de calidad, la libertad de expresión, la formación de opinión pública, las agendas informativas, la vigilancia frente al cumplimiento de la normatividad vigente, así como la creación de mecanismos de participación ciudadana para el consumo crítico de medios, entre otras cosas. En ese mismo sentido, para Antonio Roveda, los temas centrales que los observatorios latinoamericanos están trabajando actualmente son:

"Derecho a la comunicación: problemas (estudios de casos) y solicitudes de libertad de expresión, particularmente en prensa escrita.

Ética, responsabilidad social de los medios y responsabilidad periodística.

Calidad informativa de los medios, análisis de mensajes, contenidos y parrillas de programación, en especial lo referente a temas como: violencia intrafamiliar, violencia infantil, violencia política, orden público y tipos de discriminación en los mensajes de los medios.

Derecho al libre acceso a la información: equidad y equilibrio informativo; pluralismo y grado de participación en los medios, lucha contra la concentración económica, política e informativa de los medios; estudio sobre el acceso y la participación de los públicos, audiencias y de la sociedad civil en los medios, y democratización de las comunicaciones y de las tecnologías.

Estudio sobre el tratamiento de los problemas sociales de la región: discriminación por sexo, por grupo étnico y minorías; análisis de imaginarios y de referentes sociales sobre la infancia, la juventud, la tercera edad y el medio ambiente; estudios sobre el papel de los medios de comunicación en el tratamiento y la representación de los conflictos locales y continentales; análisis de informaciones y mensajes sobre lo político, y seguimiento a los discursos sociales y análisis de imágenes de paz, tratamiento de los derechos humanos; relación ( y equilibrio) entre medios de comunicación, información, elecciones políticas y acciones democráticas, y participación ciudadana, medios y educación para el consumo"14.

Existen otras experiencias, de observatorios que promueven otro tipo de escenarios más cercanos a los sectores sociales que consumen estos medios, tal es el caso de Brasil, Guatemala, Honduras, Perú y Venezuela, donde algunos observatorios se preocupan por generar algún tipo de diálogo, interacción y procesos de formación con redes de televidentes y comunicadores sobre consumo crítico.

En Colombia, sólo por mencionar una experiencia, encontramos el Observatorio de Medios de la Universidad de la Facultad de Comunicación de la Universidad de La Sabana, el cual se enfoca en tres puntos básicos: la identificación, el análisis y la discusión de la función que cumplen los medios de comunicación en Colombia, con una óptica académica que se fundamenta desde la crítica y desde la propuesta. A manera de ejemplo, el trabajo del observatorio promovió la creación del "Acuerdo por la Discreción", documento que se constituye como una herramienta fundamental referida a la responsabilidad que deben

14. BONILLA, Jorge Iván. et al. Toma el Control. Bogotá: Pontifica Universidad Javeriana. 2008, pág. 310. 


\section{Aprendizajes}

tener los medios en el cubrimiento y difusión de hechos violentos. Este observatorio se centra en la lectura crítica a los medios masivos de comunicación en su función de informar, con el fin de guiar la opinión pública y mejorar la participación que tienen los ciudadanos en las decisiones que afectan permanentemente al país.

A veces parece que la creación de un observatorio de medios es una moda, teniendo en cuenta que en América Latina existen más de cien, pero esto lo que refleja es una profunda crisis en la legitimidad de los medios tradicionales y de los discursos que transitan en ellos, probablemente esta es una de las razones por las que surge la necesidad de estudiarlos y cuestionarlos. El análisis que se produce desde estos escenarios en la mayoría de los casos tiene que ver con los contenidos, la información transmitida y cómo ésta re-produce imaginarios y concepciones de lo social, de lo cultural, de lo propio y lo foráneo, de lo individual y lo colectivo, entre otros. La meta debiera ser, primero, la resignificación de la labor propia del medio y segundo, la creación de política pública que democratice la información y potencie la implementación de los mismos en entornos micro como la escuela, dado su gran valor como institución social y como formadora de ciudadanos.

\section{La propuesta: un observatorio diferente}

El Observatorio de Medios Escolares del Distrito es importante para la Facultad de Ciencias de la Comunicación de UNIMINUTO en tanto complejiza la noción de observación y se propone como un espacio de reflexión constante (entre docentes, estudiantes e investigadores de la Universidad, pero también docentes, estudiantes de las Instituciones
Educativas participantes y otros actores sociales) que sobrepasa la noción de fiscalización y observación de los contenidos que se emiten en los medios escolares (aunque sin desconocer esta importante función) pues el proceso que subyace de las prácticas mediáticas y no mediáticas en los medios escolares se convierte en el foco de interés.

Otra característica, es que el análisis de esas prácticas que se constituyen a partir de los medios escolares, responde además a unas lógicas inscritas en el proceso enseñanza-aprendizaje o de interaprendizaje (depende el caso) propio del entorno escolar, la idea es que también esa dualidad que poseen estos medios nos permita reconocer cuál es su alcance en un ámbito semipúblico como el de la escuela, como un medio de comunicación, pero también como una herramienta para fortalecer los procesos pedagógicos propios de la institución educativa y además los formativos en

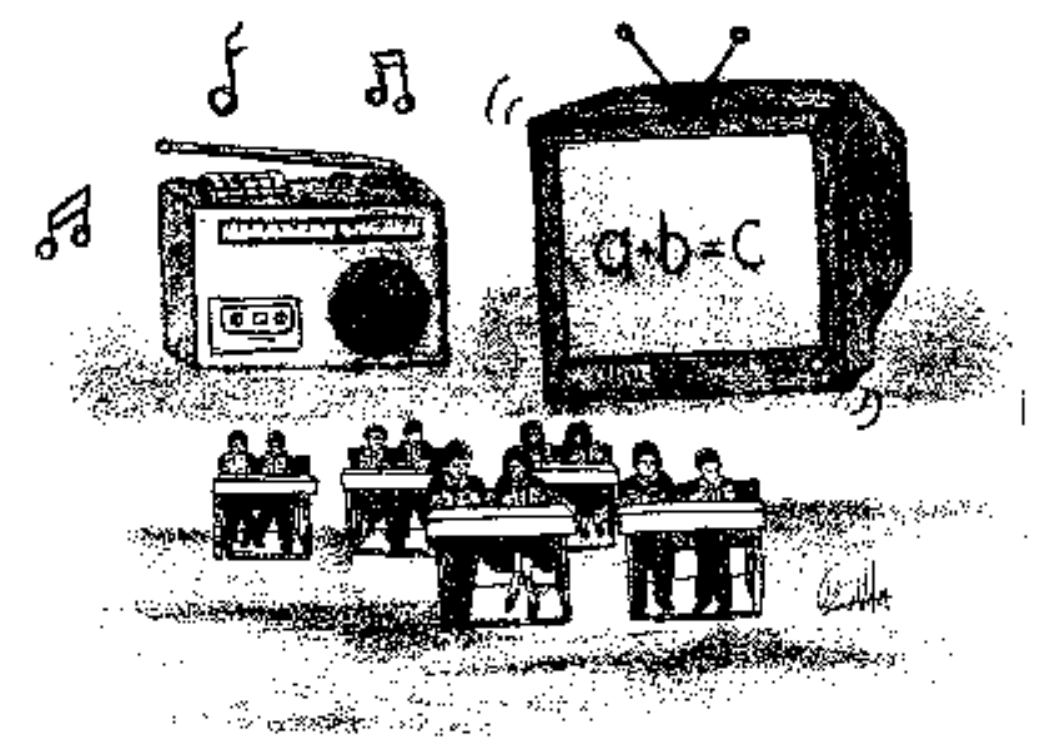


cuanto a la generación de ciudadanos preocupados por lo público, por la deliberación, por la construcción de la convivencia desde la inter-culturalidad y por la búsqueda de nuevas subjetividades desde lo político.

El Observatorio busca entonces reconocer los procesos y prácticas pedagógicas que sugieren los medios en el ámbito escolar, comprendiendo los procesos que acompañan la producción de contenidos, las relaciones que se establecen entre los diferentes agentes que componen la comunidad educativa y sus formas y niveles de incidencia en los procesos mediáticos, las motivaciones e intereses que fomentan dichas prácticas, las formas como se re-configura la estructura escolar a partir de estos procesos, en fin, las infinitas posibilidades que generan las relaciones interpersonales y los roles asumidos por los agentes involucrados en la construcción colectiva de nuevos saberes, competencias, contenidos y sentidos desde la relación comunicación-educación.

"Es sólo en la medida en que la escuela y el sistema educativo sean capaces de asumir que la tecnicidad comunicativa -que es una dimensión estratégica de cualquier cultura- forma especialmente hoy parte constitutiva de la estructura cultural de nuestra sociedad, como la escuela va a poder insertarse en la hondura de los cambios que atraviesa nuestro mundo"15.

Partiendo de lo expuesto, el Observatorio de Medios Escolares del Distrito plantea como objetivo central: generar procesos de investigación en torno a las diversas prácticas y procesos pedagógicos y comunicativos que reconozcan a los medios escolares de comunicación como una herramienta para potenciar las condiciones de desarrollo tanto de las instituciones educativas como

15. MARTíN BARBERO, op cit., pág. 18. de los contextos en que éstas se encuentran inmersas [fiel a la propuesta inicial presentada a COLCIENCIAS, pero fiel también a las necesidades del contexto de los medios escolares en Bogotá. Los objetivos específicos son básicamente cuatro: a) generar espacios de discusión y socialización de las prácticas y procesos mediáticos en entornos escolares; b) fortalecer a los colectivos de comunicación escolar y a las instituciones educativas del Distrito Capital, en términos de sus competencias analíticas sobre contexto y en el uso de los medios escolares como herramientas para el desarrollo; c) fortalecer las competencias investigativas de estudiantes de pregrado y posgrado de la Facultad de Ciencias de la Comunicación de UNIMINUTO a través de diferentes estrategias pedagógicas (semilleros de investigación, proyectos de grado, prácticas profesionales) y d) generar procesos que alimenten la construcción de una política pública de comunicación en entornos educativos.

Para lograrlo se construyó una estructura metodológica que tiene en cuenta una dimensión formativa y una transformativa que articulan los diferentes intereses y procesos tanto institucionales (Facultad de Ciencias de la Comunicación) como sociales (referidos a las dinámicas propias de las instituciones educativas y de los contextos en que están inmersas. De manera esquemática podría decirse que los elementos que constituyen cada dimensión son:

\section{Dimensión formativa:}

Proyectos de clase, con periodicidad semestral, correspondientes a los realizados desde los cursos del Área de Comunicación y Participación Ciudadana, del programa de CS-P.

Semilleros de investigación: vinculación de asistentes de investigación de pregrado y posgrado; y la realización de proyectos de grado derivados del 
proyecto macro, que contarían con la asesoría de los investigadores principales de éste.

Prácticas profesionales: vinculación de estudiantes de últimos semestres de Comunicación Social - Periodismo, interesados en la fortalecer sus competencias en investigación social, o que ven en ésta un proyecto de vida.

Fortalecimiento de los colectivos de comunicación escolar y de las instituciones educativas, tanto en sus competencias para analizar el contexto como para emplear los medios escolares como herramientas para el desarrollo.

\section{Dimensión transformativa:}

Fortalecimiento de las prácticas mediáticas en entornos escolares.

Fortalecimiento de competencias investigativas en la escuela media, pregrado y posgrado.

Generación de procesos de reflexión, cambio y mejoramiento del entorno educativo, desde el quehacer de los medios escolares de comunicación.

\section{Reflexiones finales}

Durante el periodo comprendido entre mayo del 2009 y mayo del 2010 se han realizado las siguientes actividades: Primer concurso de medios escolares, acercamiento a doce experiencias de medios escolares en el Distrito, revisión documental (posturas teóricas, investigaciones, experiencias de capacitación, legislación, convocatorias), articulación de algunas asignaturas del plan de estudios del programa de Comunicación Social - Periodismo (comunicación educativa, radio educativa y metodologías cualitativas), consolidación del semillero de investigación, realización de dos trabajos de grado y la creación del Diplomado en Medios Escolares para la Convivencia y la Ciudadanía.

\section{Aprendizajes}

Las reflexiones generales se agrupan desde tres lugares que a continuación enunciamos:

La legitimidad del medio y del proceso:

El concepto de legitimidad se relaciona con la idea de lo justo, lo que cada cual reconoce como verdadero, como propio, en términos sociales tiene que ver con los procesos de construcción de identidad y del sentido de apropiación, en la medida que los productos y los procesos corresponden a las realidades de las comunidades reconociendo sus intereses, deseos, acciones y creencias. Sobre este tema vemos que:

- Algunos medios aún no se consideran como legítimos entre los estudiantes, pues el sello institucional, para buena parte de ellos, representa una barrera, en tanto supone dominio, poder, coerción. Adicionalmente, no los ven como espacios propios, no constituyen el lugar en el que convergen sus intereses, entre otras cosas porque no existe autonomía de los estudiantes frente al medio, frente a los contenidos, frente a los formatos, es decir, su papel en la producción mediática no es protagónico.

En cuanto a la estructura organizativa del medio, hemos encontrado que si bien en el discurso pareciera que los estudiantes son los protagonistas, las prácticas evidencian grandes diferencias, pues en la mayoría de los casos siguen siendo los docentes los que determinan qué circula, quiénes pueden producir contenidos, cuáles son las agendas informativas y generan una dependencia del proceso de producción a los requerimientos académicos, específicamente a la nota.

En los diferentes procesos revisados, es común encontrar que los medios escolares se inscriben en el área de Español o Inglés, incluso en algunos 
énfasis estipulados de acuerdo con el Proyecto Educativo Institucional (PEI) de algunas instituciones, pero aún no se consideran como proyecto transversal, en tanto se desconoce su potencial frente al fortalecimiento de las competencias interpretativas, argumentativas y propositivas de los estudiantes y si se quiere, de la comunidad educativa en general. De hecho, podría pensarse que se inscribe en estas áreas por su cercanía temática, pero no porque exista claridad frente al sentido de dicha articulación.

\section{Lo pedagógico:}

En este apartado pretendemos evidenciar que esa permanente relación que debiera existir entre teoría y práctica, texto y contexto, no se hace evidente en las prácticas con los medios de comunicación escolar:

Las prácticas de la mayoría de instituciones que incluyen en su quehacer a los medios de comunicación se limitan al fortalecimiento del componente tecnológico, es decir, para la comunidad educativa prevalece la idea de que para producir contenidos con calidad lo importante es dotar al medio con tecnología de punta y su mayor afán radica justamente allí. Esta práctica genera, entre otras cosas, que el sentido del medio se reduzca a lo meramente instrumental y por ende, su capacidad de gestión y de generación de aprendizajes se asuma únicamente desde lo tecnológico. Así mismo, se evidencian nuevas exclusiones, pues a la hora de participar, los estudiantes o docentes se marginan pues sienten que no reúnen las competencias necesarias para hacer parte del medio.

- La sub-utilización del medio: esta práctica es muy común en los medios escolares, pues éstos se asumen como espacios de difusión de información, asociados al refuerzo de la identidad institucional (desde el punto de vista formal. Así, los contenidos mediáticos se limitan a la emisión sobre eventos, campañas internas, entre otras; este es el reto inicial de cualquier medio, porque se constituye en un espacio para visibilizar la vida académica o institucional, sin embargo, se pierde de vista que la información por sí sola no se constituye en un aprendizaje. De otro lado, en las experiencias que han sido consultadas, precisamente las que se destacan son aquellas que han explorado formatos y lenguajes propios de cada medio, generando nuevas oportunidades dentro de la comunidad educativa; se evidencia entonces que aquellas instituciones en donde no se profundiza en las características de cada medio, las posibilidades de potenciarlos son menores.

Uno de los elementos más significativos, hallados por algunos de los actores de las comunidades educativas consultadas, es la potencialidad que han encontrado en los medios frente el fortalecimiento de las competencias lecto-escritoras, específicamente con la prensa escolar y digital, pero también en el campo de la investigación e incluso en la exploración de nuevos recursos como las TIC en la producción de los diferentes medios. Es decir, existen instituciones donde claramente se establece una relación directa entre comunicación y procesos pedagógicos, a partir del fortalecimiento de competencias transversales como las comunicativas y argumentativas desde un aprendizaje significativo, a partir del uso de recursos como los blog, la radio, el video, etc., generando nuevos y diversos aprendizajes entre algunas asignaturas tras el aprovechamiento de las nuevas formas de circulación de saberes que permite la implementación de las TIC. 
Las prácticas culturales:

Desde los planteamientos expuestos en este documento, las prácticas culturales son fundamentales en los procesos adelantados desde los medios escolares de comunicación, bien sea para visibilizar, reconocer o negociar sentidos y perspectivas sobre lo cotidiano. Sin embargo, en el proceso adelantado encontramos bastante distancia entre los discursos y las prácticas y entre éstas y el reconocimiento de la multiculturalidad existente en los entornos escolares:

Frente al tema de la participación, pareciera que este concepto se asume únicamente desde la producción, limitando la inclusión de los estudiantes en la generación de contenidos, pero es recurrente encontrar que no se considera el medio escolar como un escenario donde se potencie la deliberación frente a los mismos, o que su quehacer pueda contribuir a la consolidación de ciudadanos preocupados por lo público; es decir, la noción de participación se reduce a "hacer parte" del equipo de producción, pero se desconocen o excluyen sus intereses y concepciones de mundo, el "hacer parte" se reduce a la capacidad operativa del equipo.

La mayoría de las experiencias revisadas, asumen las prácticas y los procesos con los medios escolares desde el ámbito de la producción de contenidos, eso está bien, pero no es suficiente frente a la complejidad de las realidades en que vivimos; es poco frecuente encontrar experiencias que articulen el trabajo de producción mediática con el análisis de lo que circula por estos medios, es decir, todo termina con la emisión de los mensajes, por eso la generación de aprendizajes desde la recepción no es común en las comunidades educativas.

\section{Aprendizajes}

Pese a lo anterior, en pocas experiencias el medio de comunicación se constituye como un elemento que fortalece la identidad, genera sentido de apropiación, y por qué no, produce y reproduce imaginarios y percepciones frente a la condición de ser joven, de pertenecer a determinada comunidad educativa, de pertenecer a un género, entre otros. Entonces, algunas experiencias mediáticas se constituyen en fuente de reconocimiento y de generación de nuevos aprendizajes individuales y colectivos.

También encontramos que hay pocas experiencias que se comprometen con la revisión o investigación de problemáticas o conflictos que se evidencian en las prácticas cotidianas de la comunidad educativa, generando desde el medio de comunicación espacios de reflexión y de participación, y de esta manera propiciar condiciones especiales para pensar el desarrollo humano y social desde los entornos educativos, lo que implica una responsabilidad institucional frente al contexto.

Como se vio a lo largo del documento, la creación del Observatorio de Medios Escolares en el Distrito Capital constituye una excelente alternativa para fortalecer las prácticas pedagógicas de las instituciones educativas del Distrito y su articulación con los procesos de formación ciudadana. Adicionalmente, plantea como reto buscar estrategias para fortalecer las competencias comunicativas de la comunidad educativa, así como la creación de una política pública que reconozca y potencie los medios escolares como posibles protagonistas de los procesos de desarrollo en la ciudad.

Para finalizar, teniendo en cuenta los planteamientos que hace la Facultad de Ciencias de la Comunicación de UNIMINUTO frente a la comunicación para el 
desarrollo (desde el punto de vista de la cultura), podría mencionarse que "la cultura se resignifica permanentemente, lo mismo que la identidad. Lo que buscamos no es tanto preservar la cultura local, regional o nacional, sino construir nuevas identidades (...) la comunicación para el desarrollo centra su atención en la construcción de nuevas regiones comunicativas. La región es aquí vista como un espacio territorial cargado de significación a partir de la interlocución entre los diferentes sujetos del desarrollo". Creemos que la propuesta aquí planteada contribuirá a los procesos impulsados desde esta mirada, en la medida que busca articular procesos pedagógicos, prácticas pedagógicas y procesos de producción mediática en entornos educativos.

\section{Bibliografía}

ALCALDÍA MAYOR, IDEP. A-prender los medios. Bogotá: Instituto para la Investigación Educativa y el Desarrollo Pedagógico. 2005.

ALCALDÍA MAYOR, SED. Comunicación y escuela: Orientaciones para la incorporación, usos y apropiación de los medios de comunicación en las Instituciones Educativas de Bogotá. Bogotá: Secretaría Educación del Distrito. 2005.

ALFARO, Rosa María. Observatorios de medios: avances, limitaciones y retos. ¿Una nueva conciencia crítica o una ruta de cambio? Lima: Calandria. 2005

ALFARO, Rosa María. "Lo educativo en la comunicación", En: La comunicación como relación para el desarrollo, Lima: Calandria. 1993, págs. 11-12.

BONILLA, Jorge Iván, et al. Toma el control. Bogotá: Pontificia Universidad Javeriana. 2008.

BONILLA, Jorge Iván, et al. Toma el Control. Bogotá: Pontifica Universidad Javeriana. 2008, pág. 310.

FACULTAD DE CIENCIASDE LACOMUNICACIÓN, UNIMINUTO. Proyecto Curricular Programa de Comunicación Social - Periodismo. Bogotá: UNIMINUTO. 2009.

FREIRE, Paulo. Pedagogía del Oprimido, Madrid: Siglo XXI Editores. 1995; PRIETO C. Daniel. La comunicación en la educación, Buenos Aires: Ed. La Crujía Ciccus. 1999.

GÓMEZ P. "Educación y comunicación: una relación conflictiva" En: Moreno y Villegas. Comunicación, educación y cultura. Relaciones, aproximaciones y nuevos retos, Bogotá: Pontificia Universidad Javeriana. 1999. 


\section{Aprendizajes}

GUTIÉRREZ, Francisco. "El Lenguaje Total. Pedagogía de los medios de comunicación". Buenos Aires: Hvmanitas. 1973. En: Jorge. Hacia una genealogía de Comunicación/Educación. Rastreo de algunos anclajes Político-Culturales. Argentina: Universidad Nacional de La Plata. 2005.

HUERGO, Jorge. Hacia una genealogía de Comunicación/ Educación. Rastreo de algunos anclajes Político-Culturales. Argentina: Universidad Nacional de La Plata. 2005.

KAPLÚN, Mario. A la educación por la comunicación: La práctica de la comunicación educativa, Chile: Unesco/Orelac. 1992.

MARTÍN-BARBERO, Jesús. "La educación en el ecosistema comunicativo", En: Revista Comunicar No. 13. 1999, págs: 13-21. En línea.

OLIVEIRA, Soares Ismar de. Sociedad de la Información o de la Comunicación. Sao Paulo: Cidade Nova, 1996.

OLIVEIRA SOARES, Ismar de. "La Educomunicación como desafío para nuestras organizaciones", En: Revista Aradü, No. 41. Quito: Ecuador.

OROZCO, Guillermo. "Desordenamientos Educativos en el Ecosistema Comunicacional" En: Comunicación Educativa en la Sociedad de la Información, Madrid: UNED. 2003.

PRIETO, Daniel. Ponencia. "Escuelay futuro". Segundo Congreso de Imagen y Pedagogía. Mazatlán: Sinaloa. 2002.

VALDERRAMA, Carlos Eduardo et. Al. ComunicaciónEducación, coordenadas abordajes, travesías, Bogotá: Universidad Central. 2000. 